\title{
EMBEDDING ORDERS INTO CENTRAL SIMPLE ALGEBRAS
}

\author{
BENJAMIN LINOWITZ AND THOMAS R. SHEMANSKE
}

\begin{abstract}
The question of embedding fields into central simple algebras $B$ over a number field $K$ was the realm of class field theory. The subject of embedding orders contained in the ring of integers of maximal subfields $L$ of such an algebra into orders in that algebra is more nuanced. The first such result along those lines is an elegant result of Chevalley [6] which says that with $B=M_{n}(K)$ the ratio of the number of isomorphism classes of maximal orders in $B$ into which the ring of integers of $L$ can be embedded (to the total number of classes) is $[L \cap \widetilde{K}: K]^{-1}$ where $\widetilde{K}$ is the Hilbert class field of $K$. Chinburg and Friedman ([7]) consider arbitrary quadratic orders in quaternion algebras satisfying the Eichler condition, and Arenas-Carmona 2] considers embeddings of the ring of integers into maximal orders in a broad class of higher rank central simple algebras. In this paper, we consider central simple algebras of dimension $p^{2}, p$ an odd prime, and we show that arbitrary commutative orders in a degree $p$ extension of $K$, embed into none, all or exactly one out of $p$ isomorphism classes of maximal orders. Those commutative orders which are selective in this sense are explicitly characterized; class fields play a pivotal role. A crucial ingredient of Chinberg and Friedman's argument was the structure of the tree of maximal orders for $S L_{2}$ over a local field. In this work, we generalize Chinburg and Friedman's results replacing the tree by the Bruhat-Tits building for $S L_{p}$.
\end{abstract}

\section{INTRODUCTION}

The subject of embedding fields and their orders into a central simple algebra defined over a number field has been a focus of interest for at least 80 years, going back to fundamental questions of class field theory surrounding the proof of the Albert-Brauer-Hasse-Noether theorem as well as work of Chevalley on matrix algebras.

To place the results of this paper in context, we offer a brief historical perspective. A major achievement of class field theory was the classification of central simple algebras defined over a number field, and the Albert-Brauer-Hasse-Noether theorem played a pivotal role in that endeavor. For quaternion algebras, this famous theorem can be stated as:

Theorem. Let $B$ be a quaternion algebra over a number field $K$, and let $L / K$ be a quadratic extension of $K$. Then there is an embedding of $L / K$ into $B$ if and only if no prime of $K$ which ramifies in $B$ splits in $L$.

Date: October 16, 2018.

2010 Mathematics Subject Classification. Primary 11R54; Secondary 11S45, $20 \mathrm{E} 42$.

Key words and phrases. Order, central simple algebra, affine building, embedding. 
The quaternion case is fairly straightforward to understand since a quaternion algebra over a field is either $2 \times 2$ matrices over the field or a (central simple) division algebra. The field extension $L / K$ is necessarily Galois, so the term splits is unambiguous.

In the general setting, we have a central simple algebra $B$ of dimension $n^{2}$ over a number field $K$. From [17] p 236, $L / K$ embeds into $B$ only if $[L: K] \mid n$, and an embeddable extension of degree $n$ is called a strictly maximal extension. The theorem above generalizes as follows. For a number field $K$, and $\nu$ any prime of $K$ (finite or infinite), let $K_{\nu}$ be the completion with respect to $\nu$ and let $B_{\nu}=B \otimes_{K} K_{\nu}$ be the local central simple algebra of dimension $n^{2}$ over $K_{\nu}$. The Wedderburn structure theorem says that $B_{\nu} \cong M_{\kappa_{\nu}}\left(D_{\nu}\right)$ where $D_{\nu}$ is a central simple division algebra of dimension $m_{\nu}^{2}$ over $K_{\nu}$, so of course $n^{2}=\kappa_{\nu}^{2} m_{\nu}^{2}$. We say that the algebra $B$ ramifies at $\nu$ iff $m_{\nu}>1$, and is split otherwise. The generalization of the classical theorem above follows from Theorem 32.15 of [18] and the corollary on p 241 of [17].

Theorem. Let the notation be as above, and suppose that $[L: K]=n$. Then there is an embedding of $L / K$ into $B$ if and only if for each prime $\nu$ of $K$ and for all primes $\mathfrak{P}$ of $L$ lying above $\nu, m_{\nu} \mid\left[L_{\mathfrak{P}}: K_{\nu}\right]$.

For example, any extension $L / K$ of degree $n$ will embed in $M_{n}(K)$ as $m_{\nu}=1$ for all $\nu$. So now we turn to the question of embedding orders into central simple algebras which is considerably more subtle. Perhaps the first important result was due to Chevalley [6].

Let $K$ be a number field, $B=M_{n}(K), L / K$ a field extension of degree $n$ and we may assume (without loss of generality from above) that $L \subset B$. Let $\mathcal{O}_{L}$ be the ring of integers of $L$. We know (see p 131 of [18]) that $\mathcal{O}_{L}$ is contained in some maximal order $\mathcal{R}$ of $B$, but not necessarily all maximal orders in $B$. Chevalley's elegant result is:

Theorem. The ratio of the number of isomorphism classes of maximal orders in $B$ into which $\mathcal{O}_{L}$ can be embedded to the total number of isomorphism classes of maximal orders is $[\widetilde{K} \cap L: K]^{-1}$ where $\widetilde{K}$ is the Hilbert class field of $K$.

In the last decade or so, there have been a number of generalizations of Chevalley's result. In 1999, Chinburg and Friedman [7] considered general quaternion algebras (satisfying the Eichler condition), but arbitrary orders $\Omega$ in the ring of integers of an embedded quadratic extension of the center, and proved a ratio of $1 / 2$ or 1 with respect to maximal orders in the algebra (though the answer is not as simple as Chevalley's). Chan and Xu [5], and independently Guo and Qin [10, again considered the quaternion algebras, but replaced maximal orders with Eichler orders of arbitrary level. Maclachlan [14] considered Eichler orders of square-free level, but replaced embeddings into Eichler orders with optimal embeddings. The first author of this paper [13] replaced Eichler orders with a broad class of Bass orders and considered both embeddings and optimal embeddings.

The first work beyond Chevalley's in the non-quaternion setting was by Arenas-Carmona [2]. The setting was a central simple algebra $B$ over a number field $K$ of dimension $n^{2}$, 
$n \geq 3$ with the proviso that the completions of $B$ (at the non-archimedean primes) have the form (in the notation above) $B_{\nu} \cong M_{\kappa_{\nu}}\left(D_{\nu}\right), n=\kappa_{\nu} m_{\nu}$, with $\kappa_{\nu}=1$ or $n$. He considered embeddings of the ring of integers $\mathcal{O}_{L}$ of an extension $L / K$ of degree $n$ into maximal orders of $B$ and proves a result analogous to Chevalley's with the Hilbert class field replaced by a spinor class field. His results come out of the theory of quadratic forms, in particular from his generalization of the notion of a spinor class field to the setting of a skew-Hermitian space over a quaternion algebra.

In this paper, we too consider generalizations beyond the quaternionic case. We consider the case where $B$ is a central simple algebra having dimension $p^{2}$ ( $p$ be an odd prime) over a number field $K$; this part of the setup is of course a special case of the one in [2]. On the other hand, like Chinburg and Friedman, we are able to describe the embedding situation for all orders $\Omega \subset \mathcal{O}_{L}$ where $L / K$ is a degree $p$ extension. As in the case of Chinburg and Friedman, the question of the proportion of the isomorphism classes of maximal orders into which $\Omega$ embeds is not simply dependent on a class field as in the case of the maximal order $\mathcal{O}_{L}$, but also on the relative discriminant (or conductor) of $\Omega$, and it is these considerations which have constrained our consideration to algebras of degree $p^{2}$.

There are other substantive differences between [2] and this work. Generalizing the ideas of [7], we are able to parametrize the isomorphism classes of maximal orders in the algebra so as to give an explicit description of those maximal orders into which $\Omega$ can be embedded, explicit enough to specify them via the local-global correspondence. Also as in [7] we are able to define the notion of a "distance ideal" associated to two maximal orders. We use this distance ideal together with the Artin map associated to $L / K$ to characterize the isomorphism classes of maximal orders into which $\Omega$ can be embedded.

Central to the arguments of Chinburg and Friedman are properties of the tree of maximal orders over a local field (the Bruhat-Tits building for $S L_{2}$ ). This paper avails itself to the structure of the affine building for $S L_{p}$, but introduces new arguments to replace those where the quaternionic case utilized the structure of the building as a tree; smaller accommodations are required since the extension $L / K$ need not be Galois as it is in the quadratic case.

One interesting observation about all the generalizations mentioned above is that class fields have played a central role. We now describe the main result. Since the question of embeddability of fields has been answered above, we presume throughout that $L / K$ is a degree $p$ extension and that $L \subset B$. Let $\mathcal{O}_{K}$ denote the ring of integers of $K$, and let $\Omega$ denote a commutative $\mathcal{O}_{K}$-order of rank $p$ in $L$, so necessarily $\Omega$ is an integral domain with field of fractions equal to $L$. It follows that $\Omega$ is contained in a maximal order $\mathcal{R}$ of $B$ (see p 131 of [18]), so we fix $\mathcal{R}$ for the remainder of this paper. Finally, we define the conductor of $\Omega$ as $\mathfrak{f}_{\Omega / \mathcal{O}_{K}}=\left\{x \in \mathcal{O}_{L} \mid x \mathcal{O}_{L} \subset \Omega\right\}$ (see [16]).

Via class field theory, we associate an abelian extension $K(\mathcal{R}) / K$ to our maximal order $\mathcal{R}$. We find that $\Omega$ embeds into all of the isomorphism classes of maximal orders except when the following two conditions are satisfied: 
(1) $L \subseteq K(\mathcal{R})$,

(2) Every prime ideal $\nu$ of $K$ which divides $N_{L / K}\left(\mathfrak{f}_{\Omega / \mathcal{O}_{K}}\right)$ splits in $L / K$.

When these two conditions hold, $\Omega$ embeds in one- $p$ th of the isomorphism classes of maximal orders, and those classes are characterized explicitly by means of the Frobenius, Frob ${ }_{L / K} \in$ $\operatorname{Gal}(L / K)$.

Following [7], an order $\Omega \subset \mathcal{R}$, but which does not embed in all maximal orders is called selective. In section 3.4 , we give examples and show that a degree $p$ division algebra admits no selective orders.

\section{LOCAL Results}

We begin with some results about orders in matrix algebras over local fields which we will need. Let $k$ be a non-archimedean local field, with unique maximal order $\mathcal{O}, V$ an $n$ dimensional vector space over $k$, and identify $\operatorname{End}_{k}(V)$ with $\mathcal{B}=M_{n}(k)$, the central simple matrix algebra over $k$. The ring $M_{n}(\mathcal{O})$ is a maximal order in $\mathcal{B}$ and can be denoted as the endomorphism ring $\operatorname{End}_{\mathcal{O}}(\mathcal{L})$, where $\mathcal{L}$ is an $\mathcal{O}$-lattice in $V$ of rank $n$. It is well known $\left((17.3)\right.$ of [18]) that every maximal order in $\mathcal{B}$ has the form $u M_{n}(\mathcal{O}) u^{-1}=\operatorname{End}_{\mathcal{O}}(u \mathcal{L})$ for some $u \in \mathcal{B}^{\times}$, and is it trivial to check the for another $\mathcal{O}$-lattice $\mathcal{M}$, we have $\operatorname{End}_{\mathcal{O}}(\mathcal{L})=\operatorname{End}_{\mathcal{O}}(\mathcal{M})$ iff $\mathcal{L}$ and $\mathcal{M}$ are homothetic: $\mathcal{L}=\lambda \mathcal{M}$ for some $\lambda \in k^{\times}$.

It is also the case that the maximal orders in $\mathcal{B}$ are in one-to-one correspondence with the vertices of the affine building associated to $S L_{n}(k)$ (see $\S 6.9$ of [1], or Chapter 19 of [9]), and so the vertices may be labeled by homothety classes of lattices in $V$, see p 148 of [3]. To realize such a labeling it is convenient to choose a basis $\left\{\omega_{1}, \ldots, \omega_{n}\right\}$ of $V$. This basis, actually the lines spanned by the basis elements, determines an apartment, and each vertex in that apartment can be identified uniquely with the homothety class of a lattice of the form $\mathcal{O} \pi^{a_{1}} \omega_{1} \oplus \cdots \oplus \mathcal{O} \pi^{a_{n}} \omega_{n}$, where $\pi$ is the local uniformizer of $k$. Since the basis and uniformizer are fixed, we shall denote this homothety class simply by $\left[a_{1}, \ldots, a_{n}\right]$, $\left(\left(a_{1}, \ldots, a_{n}\right) \in \mathbb{Z}^{n} / \mathbb{Z}(1,1, \ldots, 1)\right)$.

Let $\mathfrak{M}_{1}, \mathfrak{M}_{2}$ be two maximal orders in $\mathcal{B}=M_{n}(k)$, and write $\mathfrak{M}_{i}=\operatorname{End}_{\mathcal{O}}\left(\mathcal{L}_{i}\right)(i=1,2)$ for $\mathcal{O}$-lattices $\mathcal{L}_{i}$ in $V$. Since $\operatorname{End}_{\mathcal{O}}\left(\mathcal{L}_{i}\right)$ does not depend upon the homothety class of $\mathcal{L}_{i}$, we may assume without loss that $\mathcal{L}_{1} \subseteq \mathcal{L}_{2}$. As the lattices are both free modules over a PID, they have well-defined invariant factors: $\left\{\mathcal{L}_{2}: \mathcal{L}_{1}\right\}=\left\{\pi^{a_{1}}, \ldots, \pi^{a_{n}}\right\}$, with $a_{i} \in \mathbb{Z}$, and $a_{1} \leq \cdots \leq a_{n}$. Note that $\left\{\mathcal{L}_{1}: \mathcal{L}_{2}\right\}=\left\{\pi^{-a_{n}}, \ldots, \pi^{-a_{1}}\right\}$. Define the 'type distance' between $\mathfrak{M}_{1}$ and $\mathfrak{M}_{2}$ via the $\mathcal{L}_{i}$ to be congruence class modulo $n$ :

$$
t d_{k}\left(\mathfrak{M}_{1}, \mathfrak{M}_{2}\right)=t d_{\pi}\left(\mathfrak{M}_{1}, \mathfrak{M}_{2}\right) \equiv \sum_{i=1}^{n} a_{i} \quad(\bmod n), \text { where }\left\{\mathcal{L}_{2}: \mathcal{L}_{1}\right\}=\left\{\pi^{a_{1}}, \ldots, \pi^{a_{n}}\right\}
$$

This definition depends only on the local field, not the choice of uniformizer. The motivation for this definition comes from a consideration of how to label the vertices of a building. Those in the building for $S L_{n}(k)$ have types $0, \ldots, n-1$. Any given vertex, say the one 
corresponding to the homothety class of $\mathcal{L}$, can be assigned type 0 . Then if $\alpha \in G L_{n}(k)=$ $\mathcal{B}^{\times}$, the vertex associated to the homothety class of $\alpha \mathcal{L}$ has type congruent to $\operatorname{ord}_{\pi}(\operatorname{det} \alpha)$ $(\bmod n)($ see [19]).

\section{Maximal Orders over Number Fields}

In returning to the global setting, we recall that we are assuming that $p$ is an odd prime, and $B$ is a central simple algebra having dimension $p^{2}$ over a number field $K$. For a prime $\nu$ of $K$, we denote by $K_{\nu}$ its completion at $\nu$ and for $\nu$ a finite prime, $\mathcal{O}_{\nu}$ the maximal order of $K_{\nu}$, and $\pi=\pi_{\nu}$ a fixed uniformizer. We will denote by $J_{K}$ the idele group of $K$ and by $J_{B}$ the idele group of $B$. We denote by $n r$ the reduced norm in numerous contexts: $n r: B \rightarrow K$, $n r: B_{\nu} \rightarrow K_{\nu}$, or $n r: J_{B} \rightarrow J_{K}$, with any possible ambiguity resolved by context.

Because the degree of $B$ over $K$ is odd, $B_{\nu} \cong M_{p}\left(K_{\nu}\right)$ for every infinite prime $\nu$ of $K$, and since $p$ is prime, for any finite prime $\nu$ of $K, B_{\nu}$ is either $M_{p}\left(K_{\nu}\right)(\nu$ is said to split in $B$ ) or a central simple division algebra over $K_{\nu}$ ( $\nu$ is said to ramify in $B$ ) (see section 32 of [18]).

Given a maximal order $\mathcal{R} \subset B$, and a prime $\nu$ of $K$, we define localizations $\mathcal{R}_{\nu} \subset B_{\nu}$ by:

$$
\mathcal{R}_{\nu}= \begin{cases}\mathcal{R} \otimes_{\mathcal{O}} \mathcal{O}_{\nu} & \text { if } \nu \text { is finite } \\ \mathcal{R} \otimes_{\mathcal{O}} K_{\nu}=B_{\nu} & \text { if } \nu \text { is infinite }\end{cases}
$$

We will also be interested in the normalizers of the local orders, as well as their reduced norms. Let $\mathcal{N}\left(\mathcal{R}_{\nu}\right)$ denote the normalizer of $\mathcal{R}_{\nu}$ in $B_{\nu}^{\times}$. When $\nu$ is an infinite prime, $\mathcal{N}\left(\mathcal{R}_{\nu}\right)=B_{\nu}^{\times}$and $n r\left(\mathcal{N}\left(\mathcal{R}_{\nu}\right)\right)=K_{\nu}^{\times}$. If $\nu$ is finite, we have two cases: If $\nu$ splits in $B$, then $B_{\nu} \cong M_{p}\left(K_{\nu}\right)$ and every maximal order is conjugate by an element of $B_{\nu}^{\times}$to $M_{p}\left(\mathcal{O}_{\nu}\right)$, so every normalizer is conjugate to $G L_{p}\left(\mathcal{O}_{\nu}\right) K_{\nu}^{\times}\left(37.26\right.$ of [18]), while if $\nu$ ramifies in $B, \mathcal{R}_{\nu}$ is the unique maximal order of the division algebra $B_{\nu}\left[18\right.$, so $\mathcal{N}\left(\mathcal{R}_{\nu}\right)=B_{\nu}^{\times}$. It follows that for $\nu$ split, $\operatorname{nr}\left(\mathcal{N}\left(\mathcal{R}_{\nu}\right)\right)=\mathcal{O}_{\nu}^{\times}\left(K_{\nu}^{\times}\right)^{p}$, while for $\nu$ ramified p 153 of [18] gives that $n r\left(\mathcal{N}\left(\mathcal{R}_{\nu}\right)\right)=n r\left(B_{\nu}^{\times}\right)=K_{\nu}^{\times}$.

3.1. Type Numbers of Maximal Orders. We say that two orders $\mathcal{R}$ and $\mathcal{E}$ in $B$ are in the same genus if $\mathcal{R}_{\nu} \cong \mathcal{E}_{\nu}$ for all (finite) primes $\nu$ of $K$. By the Skolem-Noether theorem, this means they are locally conjugate at all finite primes. Denote by $\operatorname{gen}(\mathcal{R})$ the genus of $\mathcal{R}$, the set of orders $\mathcal{E}$ in $B$ which are in the same genus as $\mathcal{R}$. Again by Skolem-Noether, $\operatorname{gen}(\mathcal{R})$ is the disjoint union of isomorphism classes. The type number of $\mathcal{R}, t(\mathcal{R})$, is the number of isomorphism classes in $\operatorname{gen}(\mathcal{R})$.

By Theorem 17.3 of [18]), any two maximal orders in $B$ are everywhere locally conjugate, so the genus of maximal orders is independent of the choice of representative. So if $\mathcal{R}$ is any maximal order in $B$, the type number of $\mathcal{R}$ is simply the number of isomorphism classes of maximal orders in $B$. The question we answer is into how many of the isomorphism classes of maximal orders can an order $\Omega$ be embedded? Notice that if $\Omega$ embeds into one maximal 
order in an isomorphism class it embeds into all, since any two elements of an isomorphism class are (globally) conjugate.

Adelically, the genus of an order $\mathcal{R}$ is characterized by the coset space $J_{B} / \mathfrak{N}(\mathcal{R})$, where $\mathfrak{N}(\mathcal{R})=J_{B} \cap \prod_{\nu} \mathcal{N}\left(\mathcal{R}_{\nu}\right)$ where $\mathcal{N}\left(\mathcal{R}_{\nu}\right)$ is the normalizer of $\mathcal{R}_{\nu}$ in $B_{\nu}^{\times}$. The type number of $\mathcal{R}$ is the cardinality of the double coset space $B^{\times} \backslash J_{B} / \mathfrak{N}(\mathcal{R})$. To make use of class field theory, we need to realize this quotient in terms of the arithmetic of $K$.

Henceforth, let $\mathcal{R}$ be a maximal order in $B$. We prove

Theorem 3.1. The reduced norm on $B$ induces a bijection

$$
n r: B^{\times} \backslash J_{B} / \mathfrak{N}(\mathcal{R}) \rightarrow K^{\times} \backslash J_{K} / n r(\mathfrak{N}(\mathcal{R})) .
$$

Proof. The map is defined in the obvious way with $n r\left(B^{\times} \tilde{\alpha} \mathfrak{N}(\mathcal{R})\right)=K^{\times} n r(\tilde{\alpha}) n r(\mathfrak{N}(\mathcal{R}))$ and where $n r\left(\left(\alpha_{\nu}\right)\right)=\left(n r\left(\alpha_{\nu}\right)\right)$. We observed above that no infinite prime of $K$ ramifies in $B$, so it follows from Theorem 33.4 of [18], that $n r\left(B_{\nu}^{\times}\right)=K_{\nu}^{\times}$for all primes of $K$, including the infinite ones. Let $\tilde{a}=\left(a_{\nu}\right) \in J_{K}$, and $K^{\times} \tilde{a} \operatorname{nr}(\mathfrak{N}(\mathcal{R}))$ be an element of $K^{\times} \backslash J_{K} / n r(\mathfrak{N}(\mathcal{R}))$. We construct an idele $\tilde{\beta}=\left(\beta_{\nu}\right) \in J_{B}$ so that $B^{\times} \tilde{\beta} \mathfrak{N}(\mathcal{R}) \mapsto K^{\times} \tilde{a} n r(\mathfrak{N}(\mathcal{R}))$. For all but finitely many non-archimedean primes $\nu$ of $K, a_{\nu} \in \mathcal{O}_{\nu}^{\times}$and $\mathcal{R}_{\nu} \cong M_{p}\left(\mathcal{O}_{\nu}\right)$. Define $\beta_{\nu}$ to be the conjugate of the diagonal matrix $\operatorname{diag}\left(a_{\nu}, 1, \ldots, 1\right)$ which is contained in $\mathcal{R}_{\nu}$. For the other primes, using the local surjectivity of the reduced norm described above, let $\beta_{\nu}$ be any preimage of in $B_{\nu}^{\times}$of $a_{\nu}$. The constructed element $\tilde{\beta}$ is trivially seen to be in $J_{B}$ and given the invariance of the reduced norm under conjugation, we see that $\operatorname{nr}(\tilde{\beta})=\tilde{a}$ which establishes surjectivity.

For injectivity we first need a small claim: that the preimage of $K^{\times} n r(\mathfrak{N}(\mathcal{R}))$ under the reduced norm is $B^{\times} J_{B}^{1} \mathfrak{N}(\mathcal{R})$, where $J_{B}^{1}$ is the kernel of the reduced norm map $n r: J_{B} \rightarrow$ $J_{K}$. It is obvious that $B^{\times} J_{B}^{1} \mathfrak{N}(\mathcal{R})$ is contained in the kernel. Let $\tilde{\gamma} \in J_{B}$ be such that $n r\left(B^{\times} \tilde{\gamma} \mathfrak{N}(\mathcal{R})\right) \in K^{\times} n r(\mathfrak{N}(\mathcal{R}))$. Then $n r(\tilde{\gamma}) \in K^{\times} n r(\mathfrak{N}(\mathcal{R}))$, so write $n r(\tilde{\gamma})=k \cdot n r(\tilde{r})$ for $\tilde{r} \in \mathfrak{N}(\mathcal{R})$. Again noting that no infinite prime of $K$ ramifies in $B$, the Hasse-SchillingMaass theorem (Theorem 33.15 of [18]) implies there exists a $b \in B^{\times}$with $n r(b)=k$. Thus $n r(\tilde{\gamma})=n r(b) \cdot n r(\tilde{r})$, hence $n r\left(b^{-1}\right) \cdot n r(\tilde{\gamma}) \cdot n r\left(\tilde{r}^{-1}\right)=(1) \in J_{K}$ which implies $B^{\times} \tilde{\gamma} \mathfrak{N}(\mathcal{R})=B^{\times} b^{-1} \tilde{\gamma} \tilde{r}^{-1} \mathfrak{N}(\mathcal{R}) \in B^{\times} J_{B}^{1} \mathfrak{N}(\mathcal{R})$ as claimed.

To continue with injectivity, suppose that there exist $\tilde{\alpha}, \tilde{\beta} \in J_{B}$ with $n r\left(B^{\times} \tilde{\alpha} \mathfrak{N}(\mathcal{R})\right)=$ $n r\left(B^{\times} \tilde{\beta} \mathfrak{N}(\mathcal{R})\right)$. Then $K^{\times} n r(\tilde{\alpha}) n r(\mathfrak{N}(\mathcal{R}))=K^{\times} n r(\tilde{\beta}) n r(\mathfrak{N}(\mathcal{R}))$ which implies $n r\left(\tilde{\alpha}^{-1} \tilde{\beta}\right) \in$ $K^{\times} n r(\mathfrak{N}(\mathcal{R}))$. By the claim, we have that $\tilde{\alpha}^{-1} \tilde{\beta} \in B^{\times} J_{B}^{1} \mathfrak{N}(\mathcal{R})$. As above, it is easy to check that $B^{\times} J_{B}^{1}$ is a normal subgroup of $J_{B}$, being the kernel of the induced homomorphism $n r: J_{B} \rightarrow J_{K} / K^{\times}$, so that $\tilde{\beta} \in \tilde{\alpha} B^{\times} J_{B}^{1} \mathfrak{N}(\mathcal{R})=B^{\times} J_{B}^{1} \tilde{\alpha} \mathfrak{N}(\mathcal{R})$. By VI.iii and VII of [8], we have that $J_{B}^{1} \subset B^{\times} \tilde{\gamma} \mathfrak{N}(\mathcal{R}) \tilde{\gamma}^{-1}$ for any $\tilde{\gamma} \in J_{B}$, so choosing $\tilde{\gamma}=\tilde{\alpha}$, we have

$$
\tilde{\beta} \in B^{\times} J_{B}^{1} \tilde{\alpha} \mathfrak{N}(\mathcal{R}) \subseteq B^{\times} \tilde{\alpha} \mathfrak{N}(\mathcal{R}),
$$

so $B^{\times} \tilde{\beta} \mathfrak{N}(\mathcal{R}) \subseteq B^{\times} \tilde{\alpha} \mathfrak{N}(\mathcal{R})$, and by symmetry, we have equality. 
While it is well-known that the type number is finite (the type number of an order is trivially bounded above by its class number and the class number is finite (26.4 of [18])), we establish a stronger result in our special case of central simple algebras of dimension $p^{2}$ over $K$. We show that the type number is a power of $p$; more specifically, we show that

Theorem 3.2. Let $\mathcal{R}$ be a maximal order in a central simple algebra of dimension $p^{2}$ over a number field $K$. Then the group $K^{\times} \backslash J_{K} / n r(\mathfrak{N}(\mathcal{R}))$ is an elementary abelian group of exponent $p$.

Proof. Consider the quotient $J_{K} / n r(\mathfrak{N}(\mathcal{R}))$. Each factor in the product has the form $K_{\nu}^{\times} / \operatorname{nr}\left(\mathcal{N}\left(R_{\nu}\right)\right)$. From above, we see that this quotient is trivial when $\nu$ is infinite or finite and ramified. For finite split primes, $K_{\nu}^{\times} / n r\left(\mathcal{N}\left(R_{\nu}\right)\right)=K_{\nu}^{\times} /\left(\mathcal{O}_{v}^{\times}\left(K_{\nu}^{\times}\right)^{p}\right) \cong \mathbb{Z} / p \mathbb{Z}$. So it follows that $J_{K} / n r(\mathfrak{N}(\mathcal{R}))$ is an abelian group of exponent $p$. The canonical homomorphism $J_{K} / n r(\mathfrak{N}(\mathcal{R})) \rightarrow K^{\times} \backslash J_{K} / n r(\mathfrak{N}(\mathcal{R}))$ is surjective, so the resulting quotient is finite, abelian, and of exponent $p$ which completes the proof.

3.2. The class field associated to a maximal order. We have seen above that the distinct isomorphism classes of maximal orders in $B$ (i.e., the isomorphism classes in the genus of any given maximal order $\mathcal{R}$ ) are in one-to-one correspondence with the double cosets in the group $G=K^{\times} \backslash J_{K} / n r(\mathfrak{N}(\mathcal{R}))$. Put $H_{\mathcal{R}}=K^{\times} n r(\mathfrak{N}(\mathcal{R}))$ and $G_{\mathcal{R}}=J_{K} / H_{\mathcal{R}}$. Since $J_{K}$ is abelian, $G$ and $G_{\mathcal{R}}$ are naturally isomorphic, and since $H_{\mathcal{R}}$ contains a neighborhood of the identity in $J_{K}$, it is an open subgroup (Proposition II.6 of [11]).

Since $H_{\mathcal{R}}$ is an open subgroup of $J_{K}$ having finite index, there is by class field theory [12], a class field $K(\mathcal{R})$ associated to it. The extension $K(\mathcal{R}) / K$ is an abelian extension with $\operatorname{Gal}(K(\mathcal{R}) / K) \cong G_{\mathcal{R}}=J_{K} / H_{\mathcal{R}}$ and with $H_{\mathcal{R}}=K^{\times} N_{K(\mathcal{R}) / K}\left(J_{K(\mathcal{R})}\right)$. Moreover, a prime $\nu$ of $K$ (possibly infinite) is unramified in $K(\mathcal{R})$ if and only if $\mathcal{O}_{\nu}^{\times} \subset H_{\mathcal{R}}$, and splits completely if and only if $K_{\nu}^{\times} \subset H_{\mathcal{R}}$. Here if $\nu$ is archimedean, we take $\mathcal{O}_{\nu}^{\times}=K_{\nu}^{\times}$. From our computations at the beginning of this section, we saw that $\operatorname{nr}\left(\mathcal{N}\left(\mathcal{R}_{\nu}\right)\right)=K_{\nu}^{\times}$or $\mathcal{O}_{\nu}^{\times}\left(K_{\nu}^{\times}\right)^{p}$. In particular $K(\mathcal{R}) / K$ is an everywhere unramified extension of $K$.

Proposition 3.3. Let $S$ be any finite set of primes in $K$ which includes the infinite primes. The group $G_{\mathcal{R}}$ can be generated by cosets having representatives of the form $e_{\nu_{i}}=\left(1, \ldots, 1, \pi_{\nu_{i}}, 1, \ldots\right)$ for $\nu_{i} \notin S, \pi_{\nu_{i}}$ a uniformizer in $K_{\nu_{i}}$.

Proof. Artin reciprocity gives the exact sequence

$$
1 \longrightarrow H_{\mathcal{R}} \longrightarrow J_{K} \stackrel{\Phi}{\longrightarrow} J_{K} / H_{\mathcal{R}} \cong \operatorname{Gal}(K(\mathcal{R}) / K) \longrightarrow 1
$$

with $\Phi$ the Artin map. By the Chebotarev density theorem, there are an infinite number of primes of $K$ in the preimage of each element of $\operatorname{Gal}(K(\mathcal{R}) / K)$ under $\Phi$. The $e_{\nu_{i}}$ are the images of those primes in $J_{K}$. 
We shall denote the generators of $G_{\mathcal{R}} \cong(\mathbb{Z} / p \mathbb{Z})^{m}$ as $\left\{\bar{e}_{\nu_{i}}\right\}_{i=1}^{m}$ where the $e_{\nu_{i}}$ are the ideles of the previous proposition. Let $L / K$ be a field extension of degree $p$. We now show that the generators $\left\{\bar{e}_{\nu_{i}}\right\}$ can be chosen so that the $K$-primes $\nu_{i}$ have certain splitting properties in $L$. We shall use the symbol $(\mathfrak{P}, L / K)$ to denote the Frobenius automorphism for an unramified prime $\mathfrak{P}$ of $L$ when $L / K$ is arbitrary, but also $(\nu, L / K)$ viewed as the Artin map for a prime $\nu$ of $K$ when $L / K$ is an abelian extension.

Proposition 3.4. With the notation as above, we have:

(1) If $L \subset K(\mathcal{R})$, then we may assume that $G_{\mathcal{R}}$ is generated by elements $\left\{\bar{e}_{\nu_{i}}\right\}$ where $\nu_{i}$ splits completely in $L$ for $i>1$, and $\nu_{1}$ is inert in $L$.

(2) If $L \not \subset K(\mathcal{R})$ then we may assume that $G_{\mathcal{R}}$ is generated by elements $\left\{\bar{e}_{\nu_{i}}\right\}$ where $\nu_{i}$ splits completely in $L$ for all $i \geq 1$.

Remark 3.5. Recall that $[K(\mathcal{R}): K]=p^{m}=t(\mathcal{R})$ for $m \geq 0$. The condition $L \subset K(\mathcal{R})$ clearly forces $m \geq 1$, however when $L \not \subset K(\mathcal{R})$, it is possible that the type number equals 1 , though in that case the second part of the proposition is vacuously true.

Proof. First suppose that $L \subset K(\mathcal{R})$. Since $K(\mathcal{R}) / K$ is abelian, Galois theory provides the following exact sequence:

$$
1 \longrightarrow \operatorname{Gal}(K(\mathcal{R}) / L) \stackrel{\iota}{\longrightarrow} \operatorname{Gal}(K(\mathcal{R}) / K) \stackrel{\operatorname{res}_{L}}{\longrightarrow} \operatorname{Gal}(L / K) \longrightarrow 1 .
$$

Let $\sigma \in \operatorname{Gal}(K(\mathcal{R}) / L)$. Viewing $\operatorname{Gal}(K(\mathcal{R}) / L) \subseteq \operatorname{Gal}(K(\mathcal{R}) / K)$, we can (by Chebotarev) write $\sigma=(\nu, K(\mathcal{R}) / K)$ for an unramified prime $\nu$ of $K$. From the exact sequence, $\left.\sigma\right|_{L}=1$, but $\left.\sigma\right|_{L}=(\nu, L / K)=1$ which implies $\nu$ splits completely in $L$. Now let $\tau$ be any element of $\operatorname{Gal}(K(\mathcal{R}) / K)$ not in $\operatorname{Gal}(K(\mathcal{R}) / L)$. Writing $\tau=(\mu, K(\mathcal{R}) / K)$ ( $\mu$ unramified), we see that since $\left.\tau\right|_{L} \neq 1$, we have $(\mu, L / K) \neq 1$ which means $\mu$ does not split completely in $L$. But $L / K$ having prime degree means $\mu$ is inert in $L$. Note that for any $\tau \notin \operatorname{Gal}(K(\mathcal{R}) / L)$, $\operatorname{Gal}(K(\mathcal{R}) / K)$ is the internal direct product of $\langle\tau\rangle$ and $\operatorname{Gal}(K(\mathcal{R}) / L)$ from which the assertion follows. In particular, if $\mu$ is any prime of $K$ inert in $L, \operatorname{Gal}(K(\mathcal{R}) / K)$ is generated by $(\mu, K(\mathcal{R}) / K)$ and $\operatorname{Gal}(K(\mathcal{R}) / L)$.

Next we assume that $L \not \subset K(\mathcal{R})$; there are two cases corresponding to whether $L / K$ is Galois or not. We begin with the case that $L / K$ is Galois. Since $L / K$ has prime degree, $L \not \subset K(\mathcal{R})$ implies that $L \cap K(\mathcal{R})=K$, hence the composite extension $K(\mathcal{R}) L / L$ is abelian with $\operatorname{Gal}(K(\mathcal{R}) L / L) \cong \operatorname{Gal}(K(\mathcal{R}) / K)$ via restriction. Let $\sigma$ be any nontrivial element of $\operatorname{Gal}(K(\mathcal{R}) L / L)$, and write $\sigma=(\mathfrak{P}, K(\mathcal{R}) L / L)$ as an Artin symbol by Chebotarev, where $\mathfrak{P}$ is a prime of $L$ unramified over $K$. Put $\nu=\mathfrak{P} \cap K$. We claim that we may also assume that the inertia degree $f(\mathfrak{P} / \nu)=1$. To see this note that the set of primes of $L$ having inertia degree (over $\mathbb{Q}$ ) greater than one has density 0, and Chebotarev guarantees we may choose $\mathfrak{P}$ from a set of primes of positive density, hence the claim. Thus every nontrivial element $\sigma$ of $\operatorname{Gal}(K(\mathcal{R}) L / L)$ has the form $\sigma=(\mathfrak{P}, K(\mathcal{R}) L / L)$ with ramification index $e(\mathfrak{P} / \nu)=1$ and inertia degree $f(\mathfrak{P} / \nu)=1$, where $\nu=\mathfrak{P} \cap K$. Since $\left.(\mathfrak{P}, K(\mathcal{R}) L / L)\right|_{K(\mathcal{R})}=$ 
$(\nu, K(\mathcal{R}) / K)^{f(\mathfrak{P} / \nu)}=(\nu, K(R) / K)$, every nontrivial element of $\operatorname{Gal}(K(\mathcal{R}) / K)$ has the form $(\nu, K(\mathcal{R}) / K)$ where $\nu$ is a prime of $K$ which splits completely in $L$ as desired.

Finally, we assume $L \not \subset K(\mathcal{R})$ and $L / K$ is not Galois. Let $\widehat{L}$ denote the Galois closure of $L / K$. It is well-known (see p58 of [16]), that a prime $\nu$ of $K$ splits completely in $L$ if and only if it splits completely in $\widehat{L}$. So if we can show that $\widehat{L} \cap K(\mathcal{R})=K$, the result in this case will follow from the previous one. To that end, let $F=\widehat{L} \cap K(\mathcal{R})$. Then $K \subset F \subset K(\mathcal{R})$, so $[F: K]$ is a power of the prime $p$. Now $[L: K]=p$ implies $[\widehat{L}: K] \mid p !$, and since $F \subset \widehat{L}$, we have $[F: K] \mid p$ !. So $[F: K]=1$ or $p$. Suppose $[F: K]=p$. As $K \subset F \subset K(\mathcal{R})$, $F / K$ is an abelian extension of $K$, so in particular $F \neq L$, which implies $F \cap L=K$. Thus $F L / L$ is Galois with $\operatorname{Gal}(F L / L) \cong \operatorname{Gal}(F / K)$. In particular, $[F L: K]=p^{2}$. But $F L \subset \widehat{L}$, so $p^{2}=[F L: K]|[\widehat{L}: K]| p !$, a contradiction. Thus $\widehat{L} \cap K(\mathcal{R})=K$ as desired.

3.3. Parametrizing the isomorphism classes. Let $\mathcal{R}$ be a fixed maximal order in $B$, and recall $G_{\mathcal{R}}=J_{K} / K^{\times} n r(\mathfrak{N}(\mathcal{R})) \cong(\mathbb{Z} / p \mathbb{Z})^{m}, m \geq 0$. Let $\left\{e_{\nu_{i}}\right\}_{i=1}^{m} \subset J_{K}$ so that their images $\left\{\bar{e}_{\nu_{i}}\right\}_{i=1}^{m}$ generate $G_{\mathcal{R}}$. By Proposition 3.3 , we may choose the $\nu_{i}$ to avoid any finite set of primes; for now we simply assume that all the $\nu_{i}$ are non-archimedean and split in $B$, in particular that $B_{\nu_{i}} \cong M_{p}\left(K_{\nu_{i}}\right)$. For each $\nu_{i}$ we shall regard $\mathcal{R}_{\nu_{i}}$ as a vertex in the building for $S L_{p}\left(K_{\nu_{i}}\right)$, and let $C_{i}$ be any chamber containing $\mathcal{R}_{\nu_{i}}$. We may assume that in a given labeling of the building, $\mathcal{R}_{\nu_{i}}$ has type zero [19], and we label the remaining vertices of the chamber $C_{i}$ as $\mathcal{R}_{\nu_{i}}^{(k)}$, (having type $k$ ) $k=1, \ldots, p-1$, putting $\mathcal{R}_{\nu_{i}}^{(0)}=\mathcal{R}_{\nu_{i}}$.

Given a $\gamma=\left(\gamma_{i}\right) \in(\mathbb{Z} / p \mathbb{Z})^{m}$, we define $p^{m}$ distinct maximal orders, $D^{\gamma}$, in $B$ via the local-global correspondence by providing the following local data:

$$
\mathcal{D}_{\nu}^{\gamma}= \begin{cases}\mathcal{R}_{\nu_{i}}^{\left(\gamma_{i}\right)} & \text { if } \nu=\nu_{i} \\ \mathcal{R}_{\nu} & \text { otherwise }\end{cases}
$$

We claim that any such collection of maximal orders parametrizes the genus of $\mathcal{R}$, that is given any maximal order $\mathcal{E}$, there is a unique $\gamma \in(\mathbb{Z} / p \mathbb{Z})^{m}$, so that $\mathcal{E} \cong \mathcal{D}^{\gamma}$. To show this, let $\mathfrak{M}$ denote the set of all maximal orders in $B$, and define a map $\rho: \mathfrak{M} \times \mathfrak{M} \rightarrow G_{\mathcal{R}}$ as follows.

Let $\mathcal{R}_{1}, \mathcal{R}_{2} \in \mathfrak{M}$. For $\nu$ a finite prime of $K$ (split in $B$ ), we have defined the type distance between their localizations: $t d_{\nu}\left(\mathcal{R}_{1 \nu}, \mathcal{R}_{2 \nu}\right) \in \mathbb{Z} / p \mathbb{Z}$. For $\nu$ archimedean or $\nu$ finite and ramified in $B$, define $t d_{\nu}\left(R_{1 \nu}, R_{2 \nu}\right)=0$. Recall that since $\mathcal{R}_{1 \nu}=\mathcal{R}_{2 \nu}$ for almost all $\nu$, $t d_{\nu}\left(\mathcal{R}_{1 \nu}, \mathcal{R}_{2 \nu}\right)=0$ for almost all primes $\nu$. Let $\rho\left(\mathcal{R}_{1}, \mathcal{R}_{2}\right)$ be the image in $G_{\mathcal{R}}$ of the idele

$\left(\pi_{\nu}^{t d_{\nu}\left(\mathcal{R}_{1 \nu}, \mathcal{R}_{2 \nu}\right)}\right)$. Note that while the idele is not well-defined, its image in $G_{\mathcal{R}}$ is since the local factor at the finite split primes has the form $K_{\nu}^{\times} / \mathcal{O}_{\nu}^{\times}\left(K_{\nu}^{\times}\right)^{p}$.

We now show that any such collection of maximal orders given as the $\mathcal{D}^{\gamma}$ parametrizes the genus. 
Proposition 3.6. Let $\mathcal{R}$ be a fixed maximal order in $B$, and consider the collection of maximal orders $\mathcal{D}^{\gamma}$ defined above.

(1) If $\mathcal{E}$ is a maximal order in $B$ and $\mathcal{E} \cong \mathcal{R}$, then $\rho(\mathcal{R}, \mathcal{E})$ is trivial.

(2) If $\mathcal{E} \cong \mathcal{E}^{\prime}$ are maximal orders in $B$, then $\rho(\mathcal{R}, \mathcal{E})=\rho\left(\mathcal{R}, \mathcal{E}^{\prime}\right)$.

(3) $\mathcal{D}^{\gamma} \cong \mathcal{D}^{\gamma^{\prime}}$ if and only if $\gamma=\gamma^{\prime}$.

Proof. For the first assertion, we may assume that $\mathcal{E}=b \mathcal{R} b^{-1}$ for some $b \in B^{\times}$by SkolemNoether, which of course means $\mathcal{E}_{\nu}=b \mathcal{R}_{\nu} b^{-1}$ for each prime $\nu$. For a finite prime which splits in $B$, we may take $\mathcal{R}_{\nu}=\operatorname{End}\left(\Lambda_{\nu}\right)$ for some $\mathcal{O}_{\nu}$-lattice $\Lambda_{\nu}$, and so $\mathcal{E}_{\nu}=\operatorname{End}\left(b \Lambda_{\nu}\right)$. It follows that

$$
t d_{\nu}\left(R_{\nu}, \mathcal{E}_{\nu}\right) \equiv \operatorname{ord}_{\nu}\left(\operatorname{det}\left(b^{-1}\right)\right) \equiv \operatorname{ord}_{\nu}\left(n r\left(b^{-1}\right)\right) \quad(\bmod p),
$$

and since $G_{\mathcal{R}}$ is trivial at the archimedean primes and the finite primes which ramify in $B$, we conclude that $\rho(\mathcal{R}, \mathcal{E})=\left(\overline{n r\left(b^{-1}\right)}\right)=(\overline{1})$ in $G_{\mathcal{R}}=J_{K} / K^{\times} n r(\mathfrak{N}(\mathcal{R}))$ as $\left(n r\left(b^{-1}\right)\right)$ is in the image of $K^{\times}$in $J_{K}$.

To see the second assertion, we have as above $\mathcal{E}^{\prime}=b \mathcal{E} b^{-1}$ for some $b \in B^{\times}$and so $\mathcal{E}_{\nu}^{\prime}=b \mathcal{E}_{\nu} b^{-1}$ for each prime $\nu$. If we write $\mathcal{R}_{\nu}=\operatorname{End}\left(\Lambda_{\nu}\right)$ and $\mathcal{E}_{\nu}=\operatorname{End}\left(\Gamma_{\nu}\right)$ for $\mathcal{O}_{\nu}$-lattices $\Lambda_{\nu}$ and $\Gamma_{\nu}$, then $\mathcal{E}_{\nu}^{\prime}=\operatorname{End}\left(b \Gamma_{\nu}\right)$. Considering the elementary divisors of the lattices $\Lambda_{\nu}, \Gamma_{\nu}$ and $b \Gamma_{\nu}$, we easily see that $t d_{\nu}\left(\mathcal{R}_{\nu}, \mathcal{E}_{\nu}^{\prime}\right) \equiv t d_{\nu}\left(R_{\nu}, \mathcal{E}_{\nu}\right)+\operatorname{ord}_{\nu}\left(\operatorname{det}\left(b^{-1}\right)\right)(\bmod p)$, from which the result follows as in the first case.

For the last assertion, we need only show one direction. Fix a prime $\nu=\nu_{i}$ among the finite number used to determine the parametrization $\mathcal{D}^{\gamma}$. Then we are comparing $R_{\nu}^{\left(\gamma_{i}\right)}$ and $R_{\nu}^{\left(\gamma_{i}^{\prime}\right)}$. Since the $\mathcal{R}_{\nu}^{(k)} k=0, \ldots, p-1$ are the vertices of a fixed chamber in the affine building for $S L_{p}\left(K_{\nu}\right)$, they can be realized (p 362 of [1]) as $R_{\nu}^{(k)}=\operatorname{End}_{\mathcal{O}_{\nu}}\left(\Lambda^{(k)}\right)$ with $\Lambda^{(k)}=$ $\mathcal{O}_{\nu} \pi \omega_{1} \oplus \cdots \oplus \mathcal{O}_{\nu} \pi \omega_{k} \oplus \mathcal{O}_{\nu} \omega_{k+1} \oplus \cdots \oplus \mathcal{O}_{\nu} \omega_{p}$. Here the set $\left\{\omega_{i}\right\}$ a basis of a vector space $V / K_{\nu}$ through which we have identified $B_{\nu}=\operatorname{End}_{K_{\nu}}(V)$. It follows that $t d_{\nu}\left(R_{\nu}^{\left(\gamma_{i}\right)}, R_{\nu}^{\left(\gamma_{i}^{\prime}\right)}\right)=\gamma_{i}^{\prime}-\gamma_{i}$ $(\bmod p)$. It is now easy to see that if $\gamma \neq \gamma^{\prime}$, then $\rho\left(\mathcal{D}^{\gamma}, \mathcal{D}^{\gamma^{\prime}}\right) \neq(\overline{1})$, so $\mathcal{D}^{\gamma} \approx \mathcal{D}^{\gamma^{\prime}}$.

3.4. Selective Orders and the Main Theorem. We reestablish the notation from the introduction. Let $p$ an odd prime, $B$ a central simple algebra of dimension $p^{2}$ over a number field $K$, and $L / K$ a field extension of degree $p$ which satisfies $L \subset B$. Let $\mathcal{O}_{K}$ denote the ring of integers of $K$, and let $\Omega$ denote a commutative $\mathcal{O}_{K}$-order of rank $p$ in $L$. Necessarily $\Omega$ is an integral domain with field of fractions equal to $L$, and we have seen that $\Omega$ is contained in a maximal order $\mathcal{R}$ of $B$ which we now fix.

Given that $\Omega$ is contained in $\mathcal{R}$, the question is into which other isomorphism classes in the genus of $\mathcal{R}$ does $\Omega$ embed? Recall that since $\mathcal{R}$ is maximal, this simply asks into which isomorphism classes of maximal orders in $B$ does $\Omega$ embed? The general case is that it embeds in all the isomorphism classes, but when it does not, we follow [7] and call $\Omega$ selective. Selectivity is characterized by our main theorem. 
Theorem 3.7. With the notation fixed as above, every maximal order in $B$ contains a conjugate (by $B^{\times}$) of $\Omega$ except when the following conditions hold:

(1) $L \subseteq K(\mathcal{R})$, that is $L$ is contained in the class field associated to $\mathcal{R}$.

(2) Every prime ideal $\nu$ of $K$ which divides $N_{L / K}\left(\mathfrak{f}_{\Omega / \mathcal{O}_{K}}\right)$ splits in $L / K$.

Suppose now that both conditions (1) and (2) hold. Then precisely one-pth of the isomorphism classes of maximal orders contain a conjugate of $\Omega$. Those classes are characterized by means of the Frobenius Frob $_{L / K}$ as follows: $\mathcal{E}$ is a maximal order which contains a conjugate of $\Omega$ if and only if $\operatorname{Frob}_{L / K}(\rho(\mathcal{R}, \mathcal{E}))$ is trivial in $\operatorname{Gal}(L / K)$.

First we give some examples of selective and non-selective orders. Let $S_{\infty}$ denote the set of infinite primes of $K$ and let $\operatorname{Ram}(B)$ denote the set of primes in $K$ which ramify in $B$.

Example 3.8. Let $p$ be an odd prime, $K$ a number field with class number $p$, let $B=M_{p}(K)$, and $\mathcal{R}=M_{p}\left(\mathcal{O}_{K}\right)$. Then $G_{\mathcal{R}}=J_{K} / K^{\times}\left(J_{K} \cap\left(\prod_{\nu \notin S_{\infty}}\left(K_{\nu}^{\times}\right)^{p} \mathcal{O}_{\nu}^{\times} \prod_{\nu \in S_{\infty}} K_{\nu}^{\times}\right)\right) \cong C_{K} / C_{K}^{p} \cong$ $C_{K}$, where $C_{K}$ is the ideal class group of $K$, and $C_{K}^{p}$ the subgroup of $p$ th powers. We conclude the type number $t(\mathcal{R})=\left|G_{\mathcal{R}}\right|=p$. This means that $[K(\mathcal{R}): K]=p$ and $K(\mathcal{R}) / K$ is an everywhere unramified abelian extension of $K$, so $K(\mathcal{R}) \subset \widetilde{K}$, where $\widetilde{K}$ is the Hilbert class field of $K$. Degree considerations force $K(\mathcal{R})=\widetilde{K}$. Put $L=K(\mathcal{R})=\widetilde{K}$. Because $B$ is everywhere split, $L$ embeds into $B$. So we have $L \subseteq K(\mathcal{R}), L \subset B$. This means that $\mathcal{O}_{L}$ is selective as established in [2, [6] as well as our main theorem. Now let $\nu$ be a prime of $K$, necessarily unramified in $L=\widetilde{K}$, and consider the order $\Omega=\mathcal{O}_{K}+\nu \mathcal{O}_{L}$. We easily see that $\nu \mathcal{O}_{L} \subset \mathfrak{f}_{\Omega / \mathcal{O}_{K}}$ which implies $\mathfrak{f}_{\Omega / \mathcal{O}_{K}} \mid \nu \mathcal{O}_{L}$, hence $N_{L / K}\left(\mathfrak{f}_{\Omega / \mathcal{O}_{K}}\right) \mid N_{L / K}\left(\nu \mathcal{O}_{L}\right)=\nu^{p} \mathcal{O}_{K}$ whether $\nu$ is inert or splits completely in $L$. Since $\mathfrak{f}_{\Omega / \mathcal{O}_{K}} \neq \mathcal{O}_{L}$, we see that $\nu \mid N_{L / K}\left(\mathfrak{f}_{\Omega / \mathcal{O}_{K}}\right)$, so by condition (2) of the theorem, in the case that $\nu$ is inert, we see $\Omega$ is not selective, but when $\nu$ splits completely, $\Omega$ is selective.

Indeed, given the theorem, we have the following interesting corollary.

Corollary 3.9. Suppose there exists a field extension $L / K$ with $[L: K]=p$ which embeds into $B$, and which contains an order $\Omega \subseteq \mathcal{O}_{L}$ which is selective. Then $B \cong M_{p}(K)$. Said alternatively, suppose we are given any number field $L / K$ of degree $p$, and any suborder $\Omega \subset \mathcal{O}_{L}$. If $B$ is a degree $p$ division algebra, then $\Omega$ embeds into every maximal order in $B$ if and only if $L$ embeds into $B$. In particular, a degree $p$ division algebra admits no selective orders.

Proof. Given $L \subset B$ and $\Omega$ selective, we must have $L \subseteq K(\mathcal{R})$. Now $K(\mathcal{R})$ is the class field associated to the subgroup $H_{\mathcal{R}}=K^{\times}\left(J_{K} \cap\left[\prod_{\nu \in S_{\infty} \cup \operatorname{Ram}(B)} K_{\nu}^{\times} \times \prod_{\nu \notin S_{\infty} \cup \operatorname{Ram}(B)} \mathcal{O}_{\nu}^{\times}\left(K_{\nu}^{\times}\right)^{p}\right]\right)$. In particular, if $\nu \in \operatorname{Ram}(B)$, then $K_{\nu}^{\times} \subset H_{\mathcal{R}}$ which means that $\nu$ splits completely in the class field $K(\mathcal{R})$, hence in $L$. But this violates the Albert-Brauer-Hasse-Noether theorem which implies that no prime that ramifies in $B$ splits in $L$. 
We give the proof of the main theorem via a sequence of propositions.

Proposition 3.10. Let $\Omega$ denote an $\mathcal{O}_{K}$-order which is an integral domain whose field of fractions $L$ is a degree $p$ extension of $K$ which is contained in $B$. We assume that $\Omega$ is contained in a fixed maximal order $\mathcal{R}$ of $B$. If $L \not \subset K(\mathcal{R})$ then every isomorphism class of maximal order in $B$ contains a conjugate $\left(\right.$ by $\left.B^{\times}\right)$of $\Omega$.

Proof. Note that if the type number $t(\mathcal{R})=1$, the proposition is obviously true, so we assume $t(\mathcal{R})=[K(\mathcal{R}): K]=p^{m}$ with $m \geq 1$. By Proposition 3.4, we may choose elements $\left\{e_{\nu_{1}}, \ldots, e_{\nu_{m}}\right\} \subset J_{K}$ so that the cosets $\left\{\bar{e}_{\nu_{i}}\right\}$ generate $G_{\mathcal{R}}=J_{K} / K^{\times} n r(\mathfrak{N}(\mathcal{R}))$, and so that the primes $\nu_{i}$ of $K$ are finite and split completely in $L$. Since $[L: K]=p, L$ is a strictly maximal subfield of $B$ (section 13.1 of [17]) and consequently (Corollary 13.3 [17]), $L$ is a splitting field for $B$. We claim that all the $\nu_{i}$ are split in $B$. Fix $\nu=\nu_{i}$ and let $\mathfrak{P}$ be any prime of $L$ lying above $\nu$. As $\nu$ splits completely in $L,\left[L_{\mathfrak{P}}: K_{\nu}\right]=1$. By Theorem 32.15 of [18], $m_{\nu}$ which is the local index of $B_{\nu} / K_{\nu}$ must divide $\left[L_{\mathfrak{P}}: K_{\nu}\right]$, thus $B_{\nu} \cong M_{p}\left(K_{\nu}\right)$, as desired. Now, $L \subset B$ implies that $L \otimes_{K} K_{\nu} \cong \oplus_{\mathfrak{P} \mid \nu} L_{\mathfrak{P}} \cong K_{\nu}^{p} \hookrightarrow B \otimes_{K} K_{\nu}=B_{\nu}$. By a slight generalization of Skolem-Noether to commutative semisimple subalgebras of matrix algebras (Lemma 2.2 of [4]), we may assume we have a $K_{\nu}$-algebra isomorphism $\varphi: B_{\nu} \rightarrow M_{p}\left(K_{\nu}\right)$ such that

$\varphi(L) \subset\left(\begin{array}{cccc}K_{\nu} & & & 0 \\ & K_{\nu} & & \\ & & \ddots & \\ 0 & & & K_{\nu}\end{array}\right)$ and hence $\varphi(\Omega) \subset \varphi\left(\mathcal{O}_{L}\right) \subset\left(\begin{array}{cccc}\mathcal{O}_{\nu} & & & 0 \\ & \mathcal{O}_{\nu} & & \\ & & \ddots & \\ 0 & & & \mathcal{O}_{\nu}\end{array}\right)$. By Corollary 2.3 of [20] all maximal orders containing $\operatorname{diag}\left(\mathcal{O}_{\nu}, \ldots, \mathcal{O}_{\nu}\right)$ have a prescribed form and lie in a fixed apartment in the affine building for $S L_{p}\left(K_{\nu}\right)$ and so it follows that by a rescaling of basis we may assume in addition that $\varphi\left(\mathcal{R}_{\nu}\right)=M_{p}\left(\mathcal{O}_{\nu}\right)$.

With $\pi$ a uniformizer in $K_{\nu}$, let $\delta_{k}=\operatorname{diag}(\underbrace{\pi, \ldots, \pi}_{k}, 1, \ldots, 1) \in M_{p}\left(K_{\nu}\right), k=0, \ldots, p-1$, and define maximal orders $\mathcal{E}_{k}=\delta_{k} M_{p}\left(\mathcal{O}_{\nu}\right) \delta_{k}^{-1}$. These are all maximal orders containing $\operatorname{diag}\left(\mathcal{O}_{\nu}, \ldots, \mathcal{O}_{\nu}\right)$, and are all the vertices of a fixed chamber in the building for $S L_{p}\left(K_{\nu}\right)$. If we put $R_{\nu}^{(k)}=\varphi^{-1}\left(\mathcal{E}_{k}\right)$ for $k=0, \ldots p-1$, and $\nu \in\left\{\nu_{1}, \ldots, \nu_{m}\right\}$ we then obtain a parametrization $\mathcal{D}^{\gamma}$ of the isomorphism classes of all maximal orders in $B$ as in Equation (11). Since $\Omega \subset \mathcal{R}$, and by construction $\Omega \subset R_{\nu_{i}}^{(0)} \cap \cdots \cap R_{\nu_{i}}^{(p-1)}$ for each $\nu_{i}$, we have that $\Omega \subset \mathcal{D}_{\nu}^{\gamma}$ for all primes $\nu$ and all $\gamma$ which is to say every isomorphism class of maximal order in $B$ contains a conjugate of $\Omega$.

Next we assume that condition (1) of the theorem holds, but not condition (2). Note that since $L \subset K(\mathcal{R})$ and $K(\mathcal{R}) / K$ is an everywhere unramified abelian extension, so is $L / K$. Moreover, since $L / K$ is of prime degree (and Galois), any unramified prime splits completely or is inert. 
Proposition 3.11. Assume that $\Omega$ is an integral domain contained in $\mathcal{R}$ whose field of fractions $L \subset K(\mathcal{R})$. Assume that there is a prime $\nu$ of $K$ which divides $N_{L / K}\left(\mathfrak{f}_{\Omega / \mathcal{O}_{K}}\right)$, the norm of the conductor $\mathfrak{f}_{\Omega / \mathcal{O}_{K}}$ of $\Omega$, but which does not split completely in L. Then every isomorphism class of maximal order in $B$ contains a conjugate of $\Omega$.

Proof. Since condition (2) is assumed not to hold, we may assume by the comments above that there is a prime $\nu$ of $K$ which divides $N_{L / K}\left(\mathfrak{f}_{\Omega / \mathcal{O}_{K}}\right)$ and which is inert in $L$. Thus we may assume that $\nu \mathcal{O}_{L} \mid \mathfrak{f}_{\Omega / \mathcal{O}_{K}}$. Our first goal is to show that $\Omega \subset \mathcal{O}_{K}+\nu \mathcal{O}_{L}$.

We first assume that $\Omega$ has the form $\Omega=\mathcal{O}_{K}[a]$ for some $a \in \mathcal{O}_{L}$, and let $f$ be the minimal polynomial of $a$ over $K$. Since $\Omega \otimes_{\mathcal{O}_{K}} K \cong L, f$ is irreducible of degree $p$, and since $a$ is integral, $f \in \mathcal{O}_{K}[x]$. By Proposition 4.12 of [15], $\mathfrak{f}_{\Omega / \mathcal{O}_{K}}=f^{\prime}(a) \partial_{L / K}^{-1}=f^{\prime}(a) \mathcal{O}_{L}$ since $L / K$ everywhere unramified implies that the different $\partial_{L / K}=\mathcal{O}_{L}$. So it follows that $f^{\prime}(a) \equiv 0$ $(\bmod \nu)$. Put $\bar{a}=a+\nu \mathcal{O}_{L}$ and consider the tower of fields:

$$
O_{K} / \nu \mathcal{O}_{K} \subseteq \mathcal{O}_{K} / \nu \mathcal{O}_{K}[\bar{a}] \subseteq \mathcal{O}_{L} / \nu \mathcal{O}_{L}
$$

From top to bottom, this is a degree $p$ extension of finite fields since $\nu$ is inert in $L$, and the ring in the middle is a field since it is a finite integral domain. Since the total extension has prime degree, there are two cases.

If $\mathcal{O}_{K} / \nu \mathcal{O}_{K}[\bar{a}]=\mathcal{O}_{L} / \nu \mathcal{O}_{L}$, then $\bar{f}$ (the reduction of $f \bmod \nu \mathcal{O}_{K}$ ) is irreducible and hence is the minimal polynomial of $\bar{a}$. In particular $\bar{f}$ must be separable polynomial since finite fields are perfect. On the other hand, $\bar{f}$ and $\overline{f^{\prime}}$ share the common root $\bar{a}$, so $\bar{f}$ is not separable, a contradiction.

Thus $\mathcal{O}_{K} / \nu \mathcal{O}_{K}[\bar{a}]=\mathcal{O}_{K} / \nu \mathcal{O}_{K}$ where we view $\mathcal{O}_{K} / \nu \mathcal{O}_{K}$ embedded as usual in $\mathcal{O}_{L} / \nu \mathcal{O}_{L}$. Thus $\bar{a}=a+\nu \mathcal{O}_{L} \in \mathcal{O}_{K} / \nu \mathcal{O}_{K}$ which means that $a+\nu \mathcal{O}_{L}=b+\nu \mathcal{O}_{L}$ for some $b \in \mathcal{O}_{K}$. This means that $a \in b+\nu \mathcal{O}_{L}$ which in turn means that $\Omega=\mathcal{O}_{k}[a] \subset \mathcal{O}_{K}+\nu \mathcal{O}_{L}$.

Now consider the general case of an order $\Omega$. We show $\Omega \subset \mathcal{O}_{K}+\nu \mathcal{O}_{L}$ by showing each element of $\Omega$ is in $\mathcal{O}_{K}+\nu \mathcal{O}_{L}$. Choose $a \in \Omega$. Without loss assume $a \notin \mathcal{O}_{K}$. Then $\mathcal{O}_{K}[a]$ is an integral domain whose field of fractions is all of $L$ since $L / K$ has prime degree. Moreover, $\mathfrak{f}_{\Omega / \mathcal{O}_{K}} \mid \mathfrak{f}_{\mathcal{O}_{K}[a] / \mathcal{O}_{K}}$, so we may use the same inert prime $\nu$ for all elements of $\Omega$, and the special case now implies the general result.

By Proposition 3.4 (and its proof), we may choose primes $\nu_{1}, \ldots, \nu_{m}$ of $K$ so that the $\left\{\bar{e}_{\nu_{i}}\right\}$ generate $G_{\mathcal{R}}$, where $\nu_{i}$ splits completely in $L$ for $i>1$ and where $\nu_{1}$ is inert in $L$. Consider the situation locally at $\nu=\nu_{1}$. We have that $\Omega_{\nu} \subset \mathcal{O}_{\nu}+\nu \mathcal{O}_{L_{\nu}} \subset \mathcal{O}_{L_{\nu}}$. As in the previous proposition, we have a $K_{\nu}$-algebra isomorphism $\varphi: B_{\nu} \rightarrow M_{p}\left(K_{\nu}\right)$. Let $\mathcal{D}_{\nu}$ be a maximal order in $M_{p}\left(K_{\nu}\right)$ containing $\varphi\left(\mathcal{O}_{L_{\nu}}\right)$ and hence $\varphi(\Omega)$. Since all maximal orders in $M_{p}\left(K_{\nu}\right)$ are conjugate, writing $\mathcal{D}_{\nu}=\operatorname{End}_{\mathcal{O}_{\nu}}\left(\Lambda_{\nu}\right)$ for some $\mathcal{O}_{\nu}$-lattice $\Lambda_{\nu}$, we may assume that $\varphi$ is defined so that $\mathcal{D}_{\nu}=M_{p}\left(\mathcal{O}_{\nu}\right)$. As in the previous proposition, let $\delta_{k}=\operatorname{diag}(\underbrace{\pi, \ldots, \pi}_{k}, 1, \ldots, 1) \in$ $M_{p}\left(K_{\nu}\right), k=0, \ldots, p-1$, and define maximal orders $\mathcal{D}_{\nu}^{(k)}=\delta_{k} M_{p}\left(\mathcal{O}_{\nu}\right) \delta_{k}^{-1}$. One trivially checks that $\nu \mathcal{D}_{\nu} \in \mathcal{D}_{\nu}^{(k)}$ for $k=0, \ldots, p-1$, so that $\varphi(\Omega) \subset \varphi\left(\mathcal{O}_{\nu}+\nu \mathcal{O}_{L_{\nu}}\right) \subset \mathcal{O}_{\nu}+\nu \mathcal{D}_{\nu} \subset \mathcal{D}_{\nu}^{(k)}$ 
for $k=0, \ldots, p-1$. Putting $\mathcal{R}_{\nu}^{(k)}=\varphi^{-1}\left(\mathcal{D}_{\nu}^{(k)}\right)$, we have $\Omega \subset \mathcal{R}_{\nu}^{(k)}$ for $k=0, \ldots, p-1$, and we may use these $\mathcal{R}_{\nu}^{(k)}$ as part of the parametrization of the isomorphism classes of maximal orders. The other primes $\nu_{2}, \ldots, \nu_{m}$ all split completely in $L$, and the previous proposition shows that $\Omega$ is contained in all the local factors of our parametrization. So as before, $\Omega$ is contained in every isomorphism class of maximal order in $B$.

Finally, we assume that conditions (1) and (2) hold, and show that $\Omega$ is contained in only one-pth of the isomorphism classes of $\mathcal{R}$. We require a small technical lemma.

Lemma 3.12. As above, let $\Omega$ denote an $\mathcal{O}_{K}$-order which is an integral domain whose field of fractions $L$ is a cyclic extension of $K$ having prime degree $p$. We assume that $L$ is contained in $B$, and let $\nu$ be a prime of $K$ which is inert in $L$. If $\nu \nmid N_{L / K}\left(\mathfrak{f}_{\Omega / \mathcal{O}_{K}}\right)$, then there exists an $a \in \Omega \backslash \mathcal{O}_{K}$ so that $\nu \nmid N_{L / K}\left(\mathfrak{f}_{\mathcal{O}_{K}[a] / \mathcal{O}_{K}}\right)$.

We remark that this lemma represents a statement that in this narrow context $\Omega$ has no common non-essential discriminantal divisors, see [15], a frequent obstruction to assuming the an order has a power basis.

Proof. First note that since $\nu$ is inert in $L$, the stated condition on the conductor $\mathfrak{f}_{\Omega / \mathcal{O}_{K}}$ is equivalent to $\nu \mathcal{O}_{L} \nmid \mathfrak{f}_{\Omega / \mathcal{O}_{K}}$. Let $a \in \Omega$, and consider the tower of fields (the quotient ring in the middle being a finite integral domain):

$$
\mathcal{O}_{K} / \nu \mathcal{O}_{K} \longrightarrow\left(\mathcal{O}_{K} / \nu \mathcal{O}_{K}\right)\left[a+\nu \mathcal{O}_{L}\right] \longleftrightarrow \mathcal{O}_{L} / \nu \mathcal{O}_{L}
$$

Since $\nu$ is inert in $L,\left[\mathcal{O}_{L} / \nu \mathcal{O}_{L}: \mathcal{O}_{K} / \nu \mathcal{O}_{K}\right]=p$, so the field in the middle coincides with one of the ends. If $\left(\mathcal{O}_{K} / \nu \mathcal{O}_{K}\right)\left[a+\nu \mathcal{O}_{L}\right]=\mathcal{O}_{K} / \nu \mathcal{O}_{K}$, then $a+\nu \mathcal{O}_{L}=b+\nu \mathcal{O}_{L}$ for some $b \in \mathcal{O}_{K}$, hence $\mathcal{O}_{K}[a] \subset \mathcal{O}_{K}+\nu \mathcal{O}_{L}$. If this happens for each $a \in \Omega$, then $\Omega \subset \mathcal{O}_{K}+\nu \mathcal{O}_{L}$. Consider the conductors of these orders: Certainly, $\mathfrak{f}_{\left(\mathcal{O}_{K}+\nu \mathcal{O}_{L}\right) / \mathcal{O}_{K}} \mid \mathfrak{f}_{\Omega / \mathcal{O}_{K}}$, and $\nu \mathcal{O}_{L} \subset \mathfrak{f}_{\left(\mathcal{O}_{K}+\nu \mathcal{O}_{L}\right) / \mathcal{O}_{K}}=$ $\left\{x \in \mathcal{O}_{L} \mid x \mathcal{O}_{L} \subseteq \mathcal{O}_{K}+\nu \mathcal{O}_{L}\right\}$. But as $\nu$ is inert in $L, \nu \mathcal{O}_{L}$ is a maximal ideal, and since $\mathcal{O}_{K}+\nu \mathcal{O}_{L} \neq \mathcal{O}_{L}, \mathfrak{f}_{\left(\mathcal{O}_{K}+\nu \mathcal{O}_{L}\right) / \mathcal{O}_{K}} \neq \mathcal{O}_{L}$, so $\mathfrak{f}_{\left(\mathcal{O}_{K}+\nu \mathcal{O}_{L}\right) / \mathcal{O}_{K}}=\nu \mathcal{O}_{L}$. This implies $\nu \mathcal{O}_{L} \mid \mathfrak{f}_{\Omega / \mathcal{O}_{K}}$, a contradiction.

So there must exist an $a \in \Omega \backslash \mathcal{O}_{K}$ so that $a \notin \mathcal{O}_{K}+\nu \mathcal{O}_{L}$. This implies $\mathcal{O}_{K}[a] /\left(\nu \mathcal{O}_{L} \cap\right.$ $\left.\mathcal{O}_{K}[a]\right) \nRightarrow\left(\mathcal{O}_{K} / \nu \mathcal{O}_{K}\right)$, so we have $\mathcal{O}_{L} / \nu \mathcal{O}_{L} \cong \mathcal{O}_{K}[a] /\left(\nu \mathcal{O}_{L} \cap \mathcal{O}_{K}[a]\right)$. By Proposition 4.7 of [15], $\nu \nmid N_{L / K}\left(\mathfrak{f}_{\mathcal{O}_{K}[a] / \mathcal{O}_{K}}\right)$, as required.

Proposition 3.13. Suppose now that conditions (1) and (2) hold. Then precisely one-pth of the isomorphism classes of maximal orders in $B$ contain a conjugate of $\Omega$. Those classes are characterized by means of the Frobenius Frob $_{L / K}$ as follows: $\mathcal{E}$ is a maximal order which contains a conjugate if and only if $\operatorname{Frob}_{L / K}(\rho(\mathcal{R}, \mathcal{E}))$ is trivial in $\operatorname{Gal}(L / K)$.

Remark 3.14. First we indicate our meaning of $\operatorname{Frob}_{L / K}(\rho(\mathcal{R}, \mathcal{E}))$. Recall that $\rho(\mathcal{R}, \mathcal{E}) \in$ $G_{\mathcal{R}}=J_{K} / H_{\mathcal{R}}$, and by Artin reciprocity, there is an isomorphism $G_{\mathcal{R}} \rightarrow \operatorname{Gal}(K(\mathcal{R}) / K)$ given by the Artin map which we denote here as $\operatorname{Frob}_{K(\mathcal{R}) / K}$. Thus $\operatorname{Frob}_{k(\mathcal{R}) / K}(\rho(\mathcal{R}, \mathcal{E}))$ is an element of $\operatorname{Gal}(K(\mathcal{R}) / K)$ which we restrict to $L$. The Artin map is also compatible with restriction giving that $\left.\operatorname{Frob}_{k(\mathcal{R}) / K}(\rho(\mathcal{R}, \mathcal{E}))\right|_{L}=\operatorname{Frob}_{L / K}(\rho(\mathcal{R}, \mathcal{E}))$. 
Proof. We have assumed that $\Omega \subset \mathcal{R}$, and suppose that $\mathcal{E}$ is another maximal order in $B$. We shall show that $\mathcal{E}$ contains a conjugate of $\Omega$ if and only if $\operatorname{Frob}_{L / K}(\rho(\mathcal{R}, \mathcal{E}))$ is trivial in $\operatorname{Gal}(L / K)$. We first show that $\operatorname{Frob}_{L / K}(\rho(\mathcal{R}, \mathcal{E}))$ non-trivial in $\operatorname{Gal}(L / K)$ implies that $\mathcal{E}$ does not contain a conjugate of $\Omega$. We proceed by contradiction and assume that $\mathcal{E}$ does contain a conjugate of $\Omega$. Then there is $b \in B^{\times}$so that $\Omega \subset \mathcal{E}^{*}=b \mathcal{E} b^{-1}$. By Proposition [3.6. $\operatorname{Frob}_{L / K}(\rho(\mathcal{R}, \mathcal{E}))=\operatorname{Frob}_{L / K}\left(\rho\left(\mathcal{R}, \mathcal{E}^{*}\right)\right) \neq(\overline{1})$, so there exists a prime $\nu$ of $K$ which is inert in $L$ so that $t d_{\nu}\left(\mathcal{R}_{\nu}, \mathcal{E}_{\nu}^{*}\right) \not \equiv 0(\bmod p)$. In particular, $\mathcal{R}_{\nu}$ and $\mathcal{E}_{\nu}^{*}$ are of different types, so indeed $\mathcal{R}_{\nu} \neq \mathcal{E}_{\nu}^{*}$. View these two maximal orders as vertices in the building for $S L_{p}\left(K_{\nu}\right)$, and choose an apartment containing them. We may assume that a basis $\left\{\omega_{i}\right\}$ for the apartment is chosen in such a way that one maximal order is $\operatorname{End}_{O_{\nu}}\left(\oplus \mathcal{O}_{\nu} \omega_{i}\right)$ which we identify with $M_{p}\left(\mathcal{O}_{\nu}\right)$ and the other with $\operatorname{End}_{O_{\nu}}\left(\oplus \mathcal{O}_{\nu} \pi^{m_{i}} \omega_{i}\right)\left(0 \leq m_{1} \leq \cdots \leq m_{p}\right)$, which is identified with $\operatorname{diag}\left(\pi^{m_{1}}, \ldots, \pi^{m_{p}}\right) M_{p}\left(\mathcal{O}_{\nu}\right) \operatorname{diag}\left(\pi^{m_{1}}, \ldots, \pi^{m_{p}}\right)^{-1}=\Lambda\left(m_{1}, \ldots, m_{p}\right)=$ $\left(\begin{array}{ccccc}\mathcal{O} & \nu^{m_{1}-m_{2}} & \nu^{m_{1}-m_{3}} & \ldots & \nu^{m_{1}-m_{p}} \\ \nu^{m_{2}-m_{1}} & \mathcal{O} & \nu^{m_{2}-m_{3}} & \ldots & \nu^{m_{2}-m_{p}} \\ \nu^{m_{3}-m_{1}} & \nu^{m_{3}-m_{2}} & \ddots & \ldots & \nu^{m_{3}-m_{p}} \\ \vdots & \vdots & & \mathcal{O} & \vdots \\ \nu^{m_{p}-m_{1}} & \cdots & & \nu^{m_{p}-m_{p-1}} & \mathcal{O}\end{array}\right)$. We may assume without loss that $m_{1}=$ 0 since $\operatorname{End}(L)$ is unchanged by the homothety class of the lattice $L$, and since $\mathcal{R}_{\nu} \neq \mathcal{E}_{\nu}^{*}$, we must have that $m_{p} \geq 1$. Let $\ell$ be the smallest index so that $m_{\ell} \geq 1$. Note that the image of $M_{p}\left(\mathcal{O}_{\nu}\right) \cap \Lambda\left(m_{1}, \ldots, m_{p}\right)$ under the projection from $M_{p}\left(\mathcal{O}_{\nu}\right) \rightarrow M_{p}\left(\mathcal{O}_{\nu} / \nu \mathcal{O}_{\nu}\right)$ is contained in $\left(\begin{array}{cc}M_{\ell-1}\left(\mathcal{O}_{\nu} / \nu \mathcal{O}_{\nu}\right) & * \\ 0 & M_{p-\ell+1}\left(\mathcal{O}_{\nu} / \nu \mathcal{O}_{\nu}\right)\end{array}\right)$.

By the lemma, we can choose an element $a \in \Omega \backslash \mathcal{O}_{K}$, with $\nu \nmid N_{L / K}\left(\mathfrak{f}_{\mathcal{O}_{K}[a] / \mathcal{O}_{K}}\right)$, and since $L / K$ has prime degree, $L$ is the field of fractions of $\mathcal{O}_{K}[a]$. This allows us to invoke the Dedekind-Kummer theorem (Theorem 4.12 of [15]). Let $f$ be the minimal polynomial of $a$ over $K$. Because $L / K$ has prime degree and $a$ is integral, $f \in \mathcal{O}_{K}[x]$ and is irreducible. Since Dedekind-Kummer applies, we consider the factorization of $\bar{f} \in\left(\mathcal{O}_{K} / \nu \mathcal{O}_{K}\right)[x]$ which will mirror the factorization of $\nu$ in the field $L$. Of course we know that $\nu$ is inert, so that $\bar{f}$ is irreducible in $\left(\mathcal{O}_{K} / \nu \mathcal{O}_{K}\right)[x]$. Now since $L \subset B$, we can view $a \in B_{\nu} \cong M_{p}\left(K_{\nu}\right)$. Without loss we identify $B_{\nu}$ with the matrix algebra. Let $F$ be the characteristic polynomial of $a$ over $K_{\nu}$ which, because $a$ is integral, will have coefficients in $\mathcal{O}_{\nu}[x]$. Consider $\bar{F} \in$ $\left(\mathcal{O}_{\nu} / \nu \mathcal{O}_{\nu}\right)[x] \cong\left(\mathcal{O}_{K} / \nu \mathcal{O}_{K}\right)[x]$. Now both $\bar{f}$ and $\bar{F}$ are polynomials of degree $p$ in $\left(\mathcal{O}_{K} / \nu\right)[x]$ having $a$ for a root. We know that $\bar{f}$ is irreducible, so $\bar{f} \mid \bar{F}$, from which it follows that $\bar{F}=\bar{f}$ by degree considerations, and hence is irreducible. On the other hand, since $a \in$ $R_{\nu} \cap \mathcal{E}_{\nu}^{*}$ we know that its image under the projection from $M_{p}\left(\mathcal{O}_{\nu}\right) \rightarrow M_{p}\left(\mathcal{O}_{\nu} / \nu \mathcal{O}_{\nu}\right)$ lies in $\left(\begin{array}{cc}M_{\ell-1}\left(\mathcal{O}_{\nu} / \nu \mathcal{O}_{\nu}\right) & * \\ 0 & M_{p-\ell+1}\left(\mathcal{O}_{\nu} / \nu \mathcal{O}_{\nu}\right)\end{array}\right)$ which means that $\bar{F}$ (the characteristic polynomial of a) will be reducible over $\mathcal{O}_{\nu} / \nu \mathcal{O}_{\nu}$ by the inherent block structure, a contradiction.

Now we show the converse: Recall that $\Omega \subset \mathcal{R}$, and let $\mathcal{E}$ be another maximal order in $B$. We shall show that if $\operatorname{Frob}_{L / K}(\rho(\mathcal{R}, \mathcal{E}))$ is trivial in $\operatorname{Gal}(L / K)$ then $\mathcal{E}$ contains a 
conjugate of $\Omega$. By Proposition [3.4, we may choose primes $\nu_{1}, \ldots, \nu_{m}$ of $K$ so that the $\left\{\bar{e}_{\nu_{i}}\right\}$ generate $G_{\mathcal{R}}$, where $\nu_{i}$ splits completely in $L$ for $i>1$ and where $\nu_{1}$ is inert in $L$. Parametrize the isomorphism classes of maximal orders as in Equation (1), using $\mathcal{R}$ and in each completion $B_{\nu_{i}}$ assigning the types by using the vertices in a fixed chamber containing $\mathcal{R}_{\nu_{i}}$ in the $S L_{p}\left(K_{\nu}\right)$ building. Thus every maximal order is isomorphic to exactly one order $\mathcal{D}^{\gamma}$, for $\gamma \in(\mathbb{Z} / p \mathbb{Z})^{m}$. We have $\mathcal{R}=\mathcal{D}^{(0)}$. Let $\gamma$ be fixed with $\mathcal{E} \cong \mathcal{D}^{\gamma}$. To establish our claim, we need only show that $\Omega \subset D^{\gamma}$. By Proposition $\left[3.6, \rho(\mathcal{R}, \mathcal{E})=\rho\left(\mathcal{R}, \mathcal{D}^{\gamma}\right)\right.$ so $\operatorname{Frob}_{L / K}\left(\rho\left(\mathcal{R}, \mathcal{D}^{\gamma}\right)\right)=1$. Recall that $\mathcal{D}_{\nu}^{\gamma}=R_{\nu}$ for all $\nu \neq \nu_{i}$ and the primes $\nu_{2}, \ldots, \nu_{m}$ all split completely in $L$. Thus $\operatorname{Frob}_{L / K}\left(\rho\left(\mathcal{R}, \mathcal{D}^{\gamma}\right)\right)=\operatorname{Frob}_{L / K}\left(\nu_{1}^{t d_{\nu_{1}}\left(\mathcal{R}_{\nu_{1}}, \mathcal{D}_{\nu_{1}}^{\gamma}\right)}\right)=1$. Since Frob $_{L / K}$ has order $p$ in $\operatorname{Gal}(L / K)$, we have that $t d_{\nu_{1}}\left(\mathcal{R}_{\nu_{1}}, \mathcal{D}_{\nu_{1}}^{\gamma}\right) \equiv 0(\bmod p)$. But given that the parametrization used the vertices in a fixed chamber of the building, this is only possible if $D_{\nu_{1}}^{\gamma}=R_{\nu_{1}}$, so of course $\Omega \subset D_{\nu_{1}}^{\gamma}$. That $\Omega \subset D_{\nu_{i}}^{\gamma}$ for $i=2, \ldots, m$ follows in exactly the same way as in Proposition 3.10. Finally for $\nu \neq \nu_{i}, \Omega \subset \mathcal{R}_{\nu}=\mathcal{D}_{\nu}^{\gamma}$. Thus $\Omega \subset \mathcal{D}_{\nu}^{\gamma}$ for all primes $\nu$, and the argument is complete.

\section{REFERENCES}

1. Peter Abramenko and Kenneth S. Brown, Buildings, Graduate Texts in Mathematics, vol. 248, Springer, New York, 2008, Theory and applications. MR MR2439729

2. Luis Arenas-Carmona, Applications of spinor class fields: embeddings of orders and quaternionic lattices, Ann. Inst. Fourier (Grenoble) 53 (2003), no. 7, 2021-2038. MR MR2044166 (2005b:11044)

3. Kenneth S. Brown, Buildings, Springer-Verlag, New York, 1989. MR MR969123 (90e:20001)

4. J. Brzezinski, On two classical theorems in the theory of orders, J. Number Theory 34 (1990), no. 1, 21-32. MR MR1039764 (91d:11142)

5. Wai Kiu Chan and Fei Xu, On representations of spinor genera, Compos. Math. 140 (2004), no. 2, 287-300. MR MR2027190 (2004j:11035)

6. C. Chevalley, Algebraic number fields, L'arithmétique dan les algèbres de matrices, Herman, Paris, 1936.

7. Ted Chinburg and Eduardo Friedman, An embedding theorem for quaternion algebras, J. London Math. Soc. (2) 60 (1999), no. 1, 33-44. MR MR1721813 (2000j:11173)

8. A. Fröhlich, Locally free modules over arithmetic orders, J. Reine Angew. Math. 274/275 (1975), 112124, Collection of articles dedicated to Helmut Hasse on his seventy-fifth birthday, III. MR MR0376619 (51 \#12794)

9. Paul Garrett, Buildings and classical groups, Chapman \& Hall, London, 1997. MR 98k:20081

10. Xuejun Guo and Hourong Qin, An embedding theorem for Eichler orders, J. Number Theory 107 (2004), no. 2, 207-214. MR MR2072384 (2005c:11141)

11. P. J. Higgins, Introduction to topological groups, Cambridge University Press, London, 1974, London Mathematical Society Lecture Note Series, No. 15. MR MR0360908 (50 \#13355)

12. Serge Lang, Algebraic number theory, second ed., Graduate Texts in Mathematics, vol. 110, SpringerVerlag, New York, 1994. MR MR1282723 (95f:11085)

13. B. Linowitz, Selectivity in quaternion algebras, submitted (2010), http://arxiv.org/pdf/1005.5326.

14. C. Maclachlan, Optimal embeddings in quaternion algebras, J. Number Theory 128 (2008), 2852-2860.

15. Władysław Narkiewicz, Elementary and analytic theory of algebraic numbers, second ed., SpringerVerlag, Berlin, 1990. MR MR1055830 (91h:11107)

16. Jürgen Neukirch, Algebraic number theory, Grundlehren der Mathematischen Wissenschaften [Fundamental Principles of Mathematical Sciences], vol. 322, Springer-Verlag, Berlin, 1999, Translated from 
the 1992 German original and with a note by Norbert Schappacher, With a foreword by G. Harder. MR MR1697859 (2000m:11104)

17. Richard S. Pierce, Associative algebras, Graduate Texts in Mathematics, vol. 88, Springer-Verlag, New York, 1982, , Studies in the History of Modern Science, 9. MR MR674652 (84c:16001)

18. I. Reiner, Maximal orders, Academic Press [A subsidiary of Harcourt Brace Jovanovich, Publishers], London-New York, 1975, London Mathematical Society Monographs, No. 5. MR MR0393100 (52 \#13910)

19. Mark Ronan, Lectures on buildings, Academic Press Inc., Boston, MA, 1989. MR 90j:20001

20. Thomas R. Shemanske, Split orders and convex polytopes in buildings, J. Number Theory 130 (2010), no. 1, 101-115. MR MR2569844

Department of Mathematics, 6188 Kemeny Hall, Dartmouth College, Hanover, NH 03755

E-mail address: benjamin.linowitz@dartmouth.edu

URL: http://www.math.dartmouth.edu/ Iinowitz/

Department of Mathematics, 6188 Kemeny Hall, Dartmouth College, Hanover, NH 03755

E-mail address: thomas.r.shemanske@dartmouth.edu

URL: http://www.math.dartmouth.edu/〜 trs/ 